\title{
La modelación y las visualizaciones computarizadas en la enseñanza de la Física
}

\author{
- THE COMPUTERIZED MODELING AND VISUALIZATIONS IN TEACHING PHYSICS \\ - A MODELACIÓN E AS VISUALIZACIONES COMPUTARIZADAS NO ENSINO DA FÍSICA
}

\author{
Edgar David Guarín Castro* / edavidsg89@gmail.com \\ Harold Moreno Fernández**/ harolin84@yahoo.es
}

\begin{abstract}
Resumen
Se describe un trabajo investigativo realizado en torno al uso de herramientas informáticas, con el fin de mejorar el aprendizaje activo de sistemas complejos en Física, con estudiantes de la Licenciatura en Física de la Universidad Pedagógica Nacional. Se culminó con la implementación de una estrategia didáctica centrada en la utilización de visualizaciones computarizadas, que pretendía desarrollar Habilidades de Pensamiento Científico (HPC) en los estudiantes, resaltando la importancia de la modelización en la enseñanza de la Física. Así se cautivó la atención de los estudiantes, promoviendo visiones más elaboradas de los problemas abordados.
\end{abstract}

\section{Summary}

We describe a research conducted on the use of computer tools to enhance active learning of complex systems in physics, with undergraduate students in Physics, from National Pedagogical University. It culminated in the implementation of a teaching strategy based on the use of computerized visualizations, which sought to develop scientific thinking skills in students, emphasizing the importance of modeling in physics education. This caught the attention of students, promoting more elaborate visions of the problems addressed.

\section{Resumo}

Nós descrevemos uma pesquisa realizada sobre o uso de ferramentas de computador para melhorar a aprendizagem ativa de sistemas complexos de física, com os alunos de graduação em Física, da Universidade Pedagógica Nacional. Ela culminou com a implementação de uma estratégia de ensino baseada no uso de visualizações computadorizados, que procurou desenvolver habilidades de pensamento científico nos estudantes, enfatizando a importância da modelagem na educação física. Isso chamou a atenção dos alunos, promovendo a visões mais elaboradas dos problemas abordados.
Palabras clave

Animación, aprendizaje activo, Habilidades de Pensamiento Científico, modelización, visualización computarizada.

Keywords

Active learning, animation, computer visualization, Modeling, Scientific Thinking Skills.

Palavras chave

Animação, Aprendizagem ativo, Habilidades de Pensamento Científico, modelagem, visualização do computador.

\footnotetext{
* Licenciado en Física, Universidad Pedagógica Nacional, Bogotá, Colombia.

** Licenciado en Física, Universidad Pedagógica Nacional, Bogotá, Colombia.
} 


\section{Introducción}

Los procesos de enseñanza aprendizaje de las ciencias naturales constituyen grandes retos y desafíos para los docentes, debido a que implican pensar en las necesidades propias de cada estudiante y en las nuevas formas de aprender que están directamente involucradas con los avances tecnológicos y científicos, presentes en una sociedad globalizada y tecnificada como la nuestra.

En este sentido, el uso de las TIC's, mediado por la utilización de los computadores, ofrece a los maestros de Física la posibilidad de diseñar actividades de aprendizaje (Edelson y Reiser, 2006), en donde se logra integrar el contenido curricular con las tecnologías computacionales de las que se disponen actualmente; esto con el fin de mejorar las experiencias de los estudiantes en el aula de clase, acercándolos a la actividad científica, a fin de desarrollar Habilidades de Pensamiento Científico (HPC) para mejorar la comprensión de los conceptos que se desean enseñar.

Del mismo modo, la implementación de las TIC's en los procesos de enseñanza-aprendizaje de las ciencias naturales, en particular de la Física, permite reproducir fenómenos complejos (fenómenos con un comportamiento espacio-temporal que no es lineal), cuya construcción en el laboratorio es prácticamente imposible o cuya solución matemática es bastante tediosa, por lo que resultan poco atractivos para su estudio.

Para tratar de solucionar lo anterior, el maestro puede crear animaciones y gráficas computarizadas con las cuales se modelen algunos fenómenos naturales no lineales y de esta forma, presentarlos de una manera más agradable, que motive al estudiante a reflexionar sobre el modelo del sistema físico considerado en el momento, construyendo correlaciones entre éste y el fenómeno, para luego mejorarlo y así profundizar en la comprensión del problema.

Por esta razón, en el departamento de Física de la Universidad Pedagógica Nacional, se ha venido trabajando en la creación de modelos computacionales basados en software libre y software educativo que, por un lado le den al docente las herramientas necesarias para llevar al aula de clase problemas cada vez más complejos e interesantes, y por otro lado, propicien el aprendizaje de los temas que trata la Física en la actualidad, incentivando así a los estudiantes a observar, describir, comprender, generar ideas y predecir sobre los fenómenos que se consideran.
De este modo, dichas herramientas virtuales se constituyen como un factor relevante en la transformación y evolución de las metodologías de enseñanza, pues encaminan la labor docente hacia la creación de estrategias más eficaces y recursivas que favorezcan el desarrollo de HPC en estudiantes de diferentes niveles educativos. Así, se presenta a continuación una estrategia didáctica utilizada con estudiantes de la Licenciatura en Física de la Universidad Pedagógica Nacional. A través de dicha estrategia, se buscó desarrollar en los estudiantes Habilidades de Pensamiento Científico por medio del uso de animaciones y visualizaciones computarizadas, que simulaban situaciones físicas complejas como: el lanzamiento vertical con rozamiento del aire, el movimiento de proyectiles de gran alcance, osciladores anarmónicos y dinámica poblacional, entre otros, a partir de modelos matemáticos elaborados por medio de métodos numéricos.

\section{Herramientas tecnológicas para el mejoramiento de la enseñanza de la Física}

\section{Uso de las TIC's y desarrollo de Habilidades de Pensamiento Científico (HPC)}

Desde hace unos 40 años, el desarrollo de las HPC ha sido un tema de gran importancia para docentes y científicos, debido a la preocupación generada por los descensos en el desempeño académico de muchos estudiantes universitarios en instituciones de gran parte del mundo (Sánchez, 2002). Es así como han surgido nuevas propuestas apoyadas en la psicología cognitiva, en las teorías acerca del funcionamiento de la mente y en la utilización de herramientas computacionales para el análisis de la información; estas propuestas están dirigidas a sustentar la evolución de la nueva ciencia del conocimiento impulsada por Howard Gardner (Sánchez, 2002, Gardner, 1985), con el objetivo de ahondar en temas referentes al pensamiento, la cognición, el aprendizaje y el desarrollo humano, así como los mecanismos de construcción, extensión y aplicación del conocimiento (Sánchez, 2002).

Por esta razón y debido al gran impacto que han tenido las TIC's en las aulas de clase, resulta interesante la relación que existe entre estas y el Desarrollo de HPC, ya que por medio de las TIC's el maestro aprende a planificar, a pensar y a elaborar materiales didácticos, partiendo de la interrelación entre los aportes que brindan las Ciencias de la Educación y la Informática, 
para crear de este modo ambientes colaborativos y cooperativos en donde el estudiante pueda participar activamente en la resolución de los problemas (Ortiz y Blanco, 2009).

Sin embargo, más allá de crear estrategias didácticas innovadoras, es indispensable hacer ver al estudiante que la construcción de conocimiento es inherente al desarrollo de habilidades, las cuales permitirán que su proceso de aprendizaje sea más significativo y duradero, además de ser el promotor de una vida de mejor calidad. Por lo tanto, es importante destacar que la responsabilidad de desarrollar las HPC a través de estrategias didácticas y de promover en el estudiantado los procesos de auto-aprendizaje, recaen principalmente en el papel que juega el profesor, quien debe dar las herramientas necesarias para que sus estudiantes logren un aprendizaje autónomo con base en el diseño y la gestión de las clases.

Cabe resaltar que para cualquier tipo de estrategia educativa, las TIC's constituyen medios mas no fines, en otras palabras, son herramientas que facilitan la construcción de los contenidos disciplinares, atendiendo a los objetivos de la enseñanza y lo más importante es que conforman un instrumento que se adapta a los distintos ritmos de aprendizaje exigidos en el mundo actual. En este trabajo, el desarrollo de las HPC fue primordial, no obstante, debido a la multiplicidad de habilidades que existen, la labor se enfocó principalmente en dos tipos de HPC, que son:

1. Habilidades de nivel organizativo y analítico, relacionadas con el análisis e interpretación de datos.

2. Habilidades de nivel argumentativo y comunicativo, relacionadas con la argumentación y construcción de conclusiones (Edelson y Reiser, 2006).

Por medio de las primeras, los estudiantes analizan e interpretan los datos presentados gráficamente por la visualización; para ello es importante que el estudiante: 1 ) Identifique las variables que interactúan y que afectan el comportamiento del sistema físico simulado, a través de un modelo matemático-computacional, y 2) Construya gráficas por medio del computador a partir de los datos calculados. Por otra parte, por medio de las habilidades de nivel argumentativo y comunicativo, el estudiante logra construir y argumentar sus conclusiones ante el profesor y los demás compañeros, basándose en la interpretación realizada a las gráficas o animaciones.
Los modelos en la enseñanza de la Física: Ventajas del uso de los instrumentos computacionales

La comprensión de nuestro entorno es un trabajo arduo que pone a prueba las capacidades y habilidades intelectuales del ser humano, para sea posible describir y representar satisfactoriamente los fenómenos físicos que suelen ocurrir en nuestro universo y de los cuales tratamos de dar cuenta, a partir de las preconcepciones y la experiencia que se tenga al respecto. Por ende, se hace necesario desarrollar habilidades de pensamiento que permitan llegar a un mejor entendimiento de los fenómenos naturales y que, a su vez, brinden la oportunidad de elaborar estrategias, con las cuales el conocimiento generado pueda ser enseñado e incluso aplicado a situaciones cotidianas.

En este sentido, los modelos se constituyen como una de las herramientas de pensamiento más eficaces a la hora de estudiar el complejo mundo que conocemos, en tanto nos ayudan a racionalizar los fenómenos que observamos teniendo en cuenta sus causas y efectos (Chamizo, 2003). Desde esta perspectiva, se puede considerar a los modelos como construcciones abstractas del pensamiento humano, con las cuales es posible representar de una manera simplificada una situación, un fenómeno, un proceso, un sistema o un ente físico, a partir de sus características esenciales. Luego, los modelos tienen la misión de "describir, explicar y predecir" el comportamiento de estas entidades en diversas situaciones, de modo que sea posible profundizar y desarrollar un entendimiento adecuado acerca de las características fundamentales del fenómeno (Raviolo, 2009).

A pesar de ello, la enseñanza tradicional de la Física aborda por lo general problemas de situaciones ideales que implican la resolución de modelos matemáticos muy simplificados, los cuales aunque pueden dar una idea de los fenómenos físicos que se estudian, no son los más correctos para analizar los hechos que en realidad ocurren a nuestro alrededor. Esta situación se debe en gran parte a que los estudiantes por lo general, no poseen las herramientas matemáticas necesarias para solucionar el conjunto de ecuaciones que dan cuenta del comportamiento de un determinado sistema, complicando así su estudio en las aulas de clase.

No obstante, la utilización de los métodos numéricos implementados computacionalmente para la solución matemática de los problemas, junto con las técnicas 
de visualización, y en particular de la animación, han reducido el nivel de complejidad en el estudio de fenómenos no lineales y han aportado indudablemente, no sólo en la presentación de los resultados de los modelos propuestos, sino también en el propio análisis de predicción cuando el número de variables es muy grande, permitiendo que estos problemas se puedan presentar en cursos cada vez más elementales de Física.

Con base en lo anterior, el maestro puede acercar a los estudiantes a situaciones más reales e interesantes por medio de modelos físicos, los cuales, hablando desde un punto de vista psicológico, actúan como intermediarios entre el individuo y su mundo, posibilitando su comprensión, su interpretación y su actuación en él (Johnson-Laird, 1996). Aquí cabe aclarar que, como afirma Blanchard et. al., (1999) con estos modelos no se busca "producir una copia exacta del objeto real, sino más bien representar algunas características de la cosa real", teniendo en cuenta el proceso de modelación matemática de fenómenos naturales, de modo que el estudiante interiorice el papel que juega la Matemática en la Física, como eje de articulación entre los sistemas reales objeto de estudio y la búsqueda de explicaciones cualitativas y cuantitativas subyacentes en el quehacer del científico.

De este modo se pretende que el futuro docente construya sus propias herramientas computacionales, motivándose a llevar modelos nuevos, sencillos o complejos, al aula de clase, haciendo uso de los métodos numéricos para la solución de las ecuaciones diferenciales que caracterizan los sistemas complejos de la Física. Gracias a la sencillez de los algoritmos, estos pueden ser enseñados incluso en cursos introductorios de Física, a diferencia de los algoritmos analíticos de solución de ecuaciones diferenciales, los cuales deben tener un fuerte cimiento matemático y cuya aplicabilidad está limitada sólo a sistemas lineales y algunos pocos no lineales.

\section{Metodología de trabajo}

La metodología de trabajo se desarrolló con 33 estudiantes de octavo y noveno semestre de la Licenciatura en Física de la Universidad Pedagógica Nacional, siguiendo una investigación cualitativa interactiva. La modelación de los fenómenos no lineales abordados en clase, se fundamentó en un estudio descriptivo realizado a lo largo de las siguientes fases:
1. Fase preliminar. En ella se realiza una indagación sobre los conceptos previos de los estudiantes para conocer cuáles son las herramientas conceptuales y matemáticas de las que dispone, a fin de escoger adecuadamente los modelos físicos a simular y el nivel de complejidad de los mismos. Se realizaron las siguientes preguntas para indagar sobre los conceptos previos de los estudiantes.

a. ¿Qué entiende por sistema físico no lineal?

b. ¿Qué métodos conoce para la resolución de ecuaciones diferenciales no lineales?

c. ¿Sabe qué es un método numérico, conoce alguno?

d. ¿Qué condiciones o parámetros tiene en cuenta para describir el comportamiento de un sistema?

e. ¿Tiene conocimientos en programación? Si_ No_

f. ¿Qué lenguaje de programación ha utilizado?

g. ¿Qué aspectos de la programación maneja? (marque con una $\mathrm{x}$ )

- Definición de funciones.

- Manejo de condicionales.

- Manejo de variables.

- Manejo de arreglos.

- Manejo de datos y gráficos.

2. Fase de construcción del modelo. Luego de escoger un fenómeno físico viable e interesante, se procede a realizar una investigación teórica sobre el mismo, con la cual se sustente el modelo computacional a desarrollar. Esto permite poner en contexto la actividad de modelamiento que se desarrollará, para después establecer la estructura general del modelo y las operaciones analíticas que favorecerán su construcción.

\section{Fase de implementación y visualización del mo-} delo. Los estudiantes, con la guía del profesor, construyen representaciones visuales del modelo como gráficas y animaciones computarizadas, uti- 


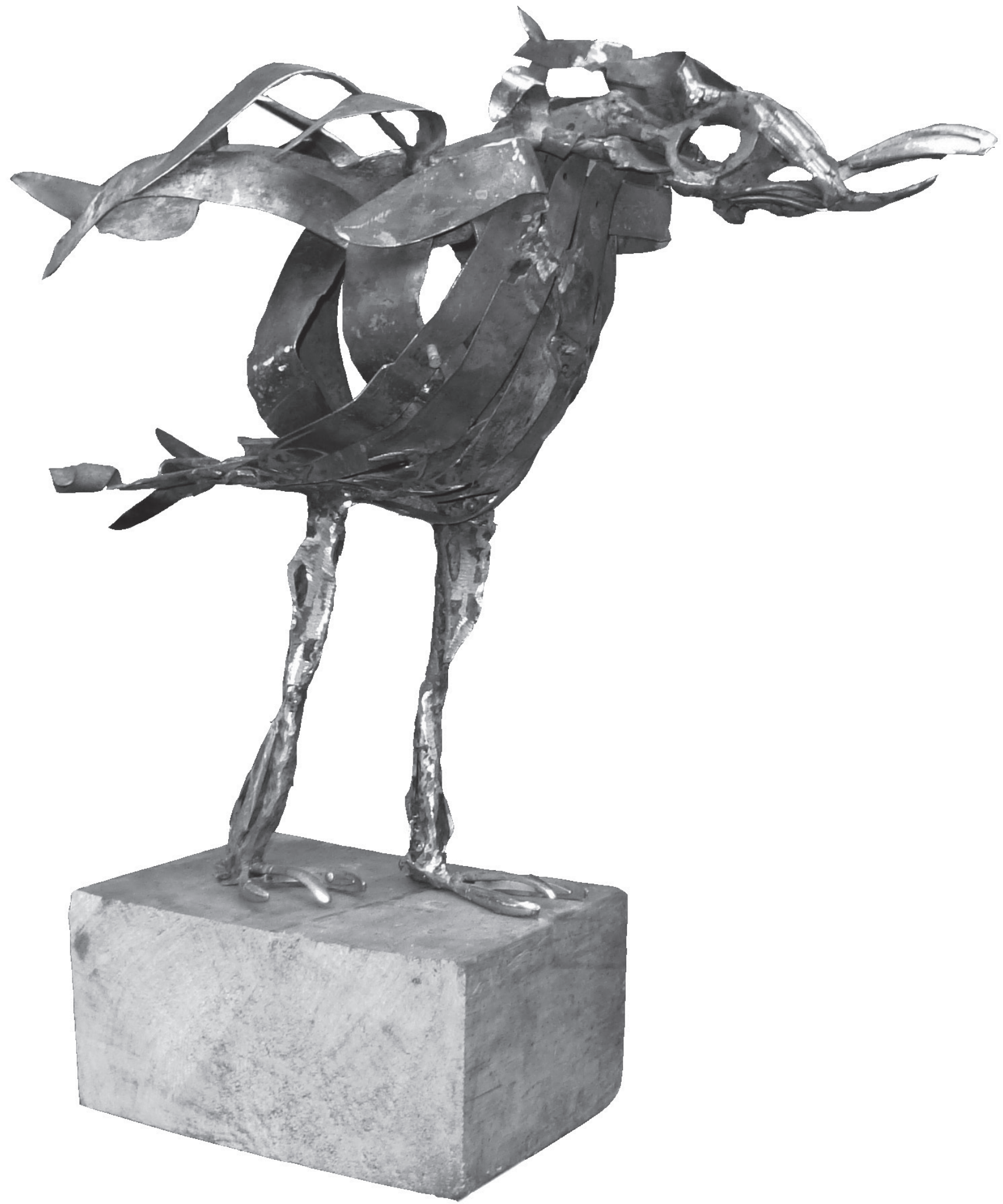

Artista: Felipe Ruiz

Titulo: Homenaje a Obregon

Dimensiones: $55 \times 45 \times 25 \mathrm{~cm}$

Tecnica: Escultura en metal

Año: 2011 
lizando los programas gnuplot y PovRay respectivamente, los cuales se vinculan a códigos numéricos elaborados en el lenguaje de programación $\mathrm{C}++$. Dichas visualizaciones deberán ser validadas con las situaciones que se esperaban y con posibles datos experimentales que se encontrarán en fuentes de información; con ello los estudiantes podrán analizar e interpretar mejor los datos y expresar su entendimiento actual acerca de los fenómenos que en ocasiones resulten poco familiares (Edelson y Reiser, 2006).

4. Fase de socialización. Los estudiantes construyen interpretaciones y conclusiones sobre el modelo para dar explicación a las situaciones obtenidas, comparando puntos de vista con el profesor y los demás compañeros, para luego reflexionar y obtener una mejor comprensión del fenómeno. Esto se propicia a través de la realización de exposiciones, discusiones en clase y en la elaboración de trabajos escritos e informes.

\section{Matriz de valoración de las Habilidades de}

\section{Pensamiento Científico}

Se hizo uso de la siguiente matriz de valoración para determinar el impacto de las actividades propuestas, en el desarrollo de las HPC en los estudiantes.

\section{Análisis e interpretación de datos}

- Resuelve y analiza situaciones problemáticas.

- Realiza relaciones, transformaciones y establece consecuencias, a partir de los datos y gráficos obtenidos.

- Obtiene información implícita para utilizarla como parte de los datos que permiten interpretar el fenómeno.

- Establece relaciones entre las hipótesis y las representaciones gráficas en dos y tres dimensiones.

- Determina el grado de credibilidad que se debe otorgar a una fuente de información o a una opinión.

\section{Construcción y justificación de conclusiones}

- Es capaz de comunicar hipótesis, resultados, planes e ideas.
- Utiliza la información recolectada para emitir y justificar conclusiones sobre los fenómenos estudiados.

- Utiliza diversos métodos para lograr comunicar sus conclusiones, tales como, escritos, visualizaciones computarizadas, diagramas y/o expresión oral.

- Sabe cuándo transferir y generalizar conclusiones, resultados, conocimientos, procesos, etcétera.

- Utiliza ejemplos, contraejemplos y/o analogías como fuentes de aprendizaje.

- Aporta elementos persuasivos para tratar de convencer al grupo y orientar los acuerdos colectivos en el salón de clases.

- Emplea con éxito varias formas de razonamiento: analógico, aritmético, dialéctico, etc.

- Determina cuáles conclusiones, de varias posibles, están mejor apoyadas o confirmadas por la evidencia disponible, o cuáles deben ser rechazadas o consideradas como menos plausibles.

\section{Resultados}

El tiempo empleado para la elaboración de cada modelo fue de dos semanas. En todos los casos se trabajó con los estudiantes según las fases mencionadas con anterioridad, sin perder de vista el objetivo principal que era desarrollar las HPC mencionadas en la sección 2.1. El aprendizaje de los estudiantes fue evaluado a través de las observaciones hechas en las clases, de la matriz de valoración presentada en el apartado anterior, de las exposiciones y discusiones llevadas a cabo en el aula y de los informes elaborados y entregados al finalizar el desarrollo de cada uno de los modelos físicos.

De las preguntas de recolección de conceptos previos

A continuación se presenta en la Tabla 1, el porcentaje de respuestas a las preguntas e y $g$, relacionadas con la recolección de conceptos previos. Las demás preguntas permitieron apreciar el nivel de proximidad que los estudiantes tenían en relación con los temas que se querían abordar. 


\begin{tabular}{|c|c|c|}
\hline \multicolumn{2}{|c|}{ ¿Tiene conocimientos en programación? } \\
\hline SI 25 estud. & NO & estud. \\
\hline \multicolumn{2}{|c|}{ ¿Qué aspectos de programación maneja? } \\
\hline Ítem & Porcentaje (de 25 estudiantes) \\
\hline Definición de funciones & $20 \%$ \\
\hline Manejo de condicionales & $90.9 \%$ \\
\hline Manejo de variables & $100 \%$ \\
\hline Manejo de arreglos & $36.36 \%$ \\
\hline Manejo de datos y gráficos & $51.51 \%$ \\
\hline
\end{tabular}

Tabla 1. Resultados a las preguntas e y g, hechas a 33 estudiantes

Los porcentajes presentados en la Tabla 1 para la pregunta g., fueron calculados a partir de las respuestas de los 25 estudiantes que contestaron afirmativamente a la pregunta e. Con base en lo anterior, se presenta a continuación una síntesis de los modelos desarrollados y las actividades realizadas para propiciar el desarrollo de HPC en los estudiantes.

\section{De los modelos físicos desarrollados por medio del uso de recursos tecnológicos}

En la actualidad existen gran cantidad de recursos tecnológicos que permiten crear modelos físicos de una manera sencilla y con grandes ventajas para los docentes, ya que les permiten elaborar estrategias didácticas haciendo uso de hojas de cálculo como Excel o de software más especializado como Matlab. Desafortunadamente, este tipo de software que posee además una muy buena precisión en la animación, implica grandes costos para los maestros o las instituciones donde se quieren implementar.

Por este motivo, se quiso aportar en la producción de material didáctico para la enseñanza de la Física, creando códigos de programación modificables en lenguaje $\mathrm{C}++\mathrm{y}$ animaciones elaboradas con gran detalle usando PovRay, buscando que el futuro docente pueda interactuar y llevar al aula las animaciones producidas. La escogencia de este software radica principalmente en que su licencia es libre, por lo que se puede encontrar y descargar fácilmente desde la Internet.
A partir de lo anterior, se desarrollaron con los estudiantes una serie de códigos numéricos, con los cuales se simularon algunas situaciones físicas complejas. El análisis de estos problemas se fundamentó en el estudio de sistemas no lineales, a partir de la resolución de las ecuaciones de movimiento, haciendo uso de métodos numéricos como el de medio punto y el de Runge Kutta de $4^{\circ}$ orden (RK 4).

Para la construcción de cada modelo, se siguió la metodología de trabajo planteada en el apartado anterior, buscando que los estudiantes fueran los agentes centrales en el proceso de elaboración de las simulaciones, acompañados siempre de la guía del profesor; esto con el propósito de generar el puente entre el entendimiento corriente que tenían los estudiantes sobre el fenómeno y un entendimiento más avanzado sobre el mismo, que les permitiera manipular y revisar las representaciones que poseían (Edelson y Reiser, 2006).

En una primera instancia se crearon modelos con los que se obtuvieron gráficas en dos y tres dimensiones con ayuda del graficador gnuplot, en las que se apreciaba el comportamiento espacio-temporal del sistema en consideración. Dichas gráficas comprendían diagramas de fase, de Posición vs. Tiempo, de Velocidad vs. Tiempo, entre otras. Aquí se destacan, entre muchos otros, los modelos creados y analizados por los estudiantes para los siguientes sistemas: 
1. Lanzamiento vertical con rozamiento del aire. Debido a que esta situación implica considerar en la ecuación de movimiento, términos con potencias al cuadrado de la velocidad, como en la ecuación (1), con los que se representa la fuerza de fricción del aire.

$$
\vec{F}_{r}=-c v^{2}
$$

(Siendo c una constante de fricción dependiente de la forma del cuerpo y de las características del aire),

\section{Ecuación1}

El estudio matemático de esta situación requiere de herramientas del cálculo que muchas veces los estudiantes de cursos introductorios de Física no poseen o no manejan muy bien, por lo que se hace necesario la utilización de métodos numéricos basados en ecuaciones algebraicas (González y Hernández, 2008), que permitan reducir la complejidad del análisis matemático. De este modo se construyó un código que resuelve la ecuación del movimiento del sistema a partir del método numérico de medio punto, para luego graficar las soluciones (Figura 1).

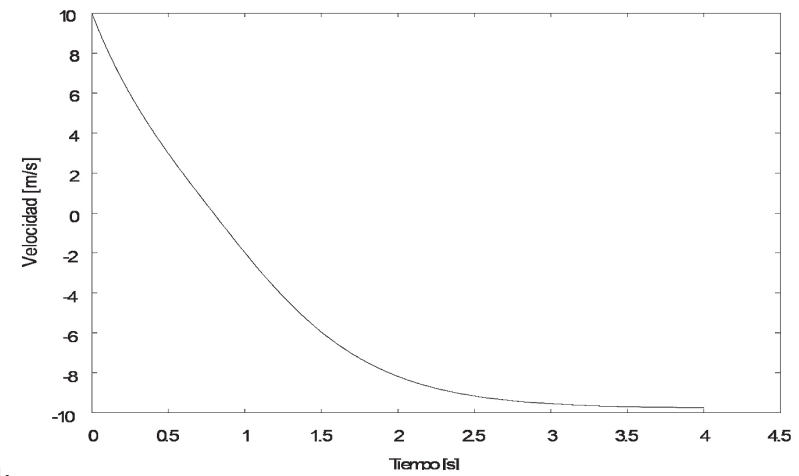

a.

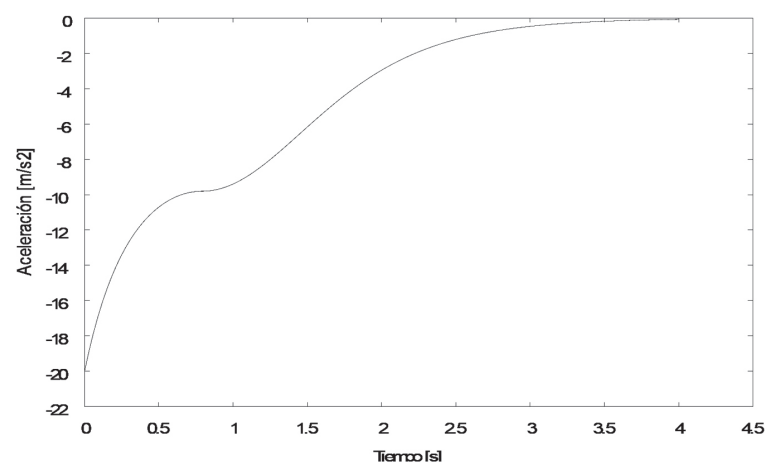

Figura 1. a. Variación de la velocidad en el tiempo de una pelota en lanzamiento vertical; b. Aceleración del movimiento de lanzamiento vertical de una pelota. Ambas graficadas por métodos numéricos con el programa Gnuplot
La verificación del modelo se hizo comparando las tablas de datos y las gráficas obtenidas con otros modelos desarrollados con métodos numéricos distintos y que se pueden encontrar en la literatura como por ejemplo en González y Hernández (2008). Esto permitió establecer que el modelo estaba acorde con lo esperado, por lo que puede ser utilizado para estudiar el sistema con otro tipo de condiciones iniciales y de frontera.

2. Oscilador de Atwood. Este es un sistema que consiste en una polea de masa $m_{d}$ y radio RR $R$, que gira en torno a un eje que pasa por su centro (Franco, 2008); sobre el disco de la polea se pega una masa $m$ a una distancia $\pi r r$ de su centro y se equilibra el sistema colgando una masa $M$ de una cuerda enrollada al disco (Figura 2)

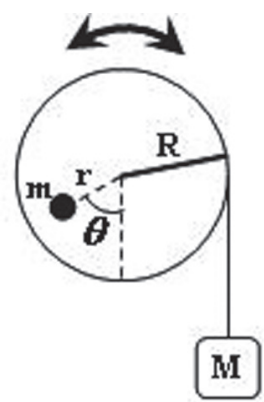

Figura 2. Esquema del montaje para un oscilador de Atwood

Teniendo en cuenta este montaje, los estudiantes Ilegaron a las ecuaciones de movimiento de las masas y elaboraron un código numérico que permitía obtener las gráficas con las que lograron describir el comportamiento del sistema para diferentes situaciones (Figura 3).

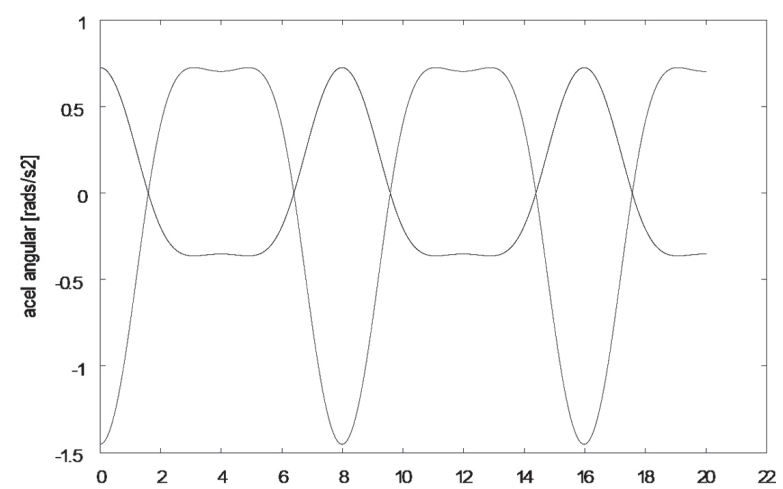

Figura 3. Variación de la aceleración angular en el tiempo para la masa m (rojo) y para la masa M (verde)

3. Dinámica poblacional. Teniendo como referente el artículo "Modelos dinámicos de guerra: 
El conflicto colombiano" (Isaza y Campos, 2005), se propuso a los estudiantes que desarrollaran un modelo usando el método numérico de Runge Kutta de $4^{\circ}$ orden, que permitiera describir cómo sería el comportamiento de dos ejércitos (Ejército y Guerrilla), enfrentados en un conflicto armado. Los estudiantes, apoyándose en las ecuaciones diferenciales presentadas en el artículo anteriormente citado, implementaron el método RK 4 para solucionarlas y obtuvieron el espacio de fase de este sistema (Figura 4), con el cual pudieron determinar cómo varía en el tiempo el número de efectivos de ambos ejércitos y cuál sería el destino de una guerra con las características planteadas en este modelo.

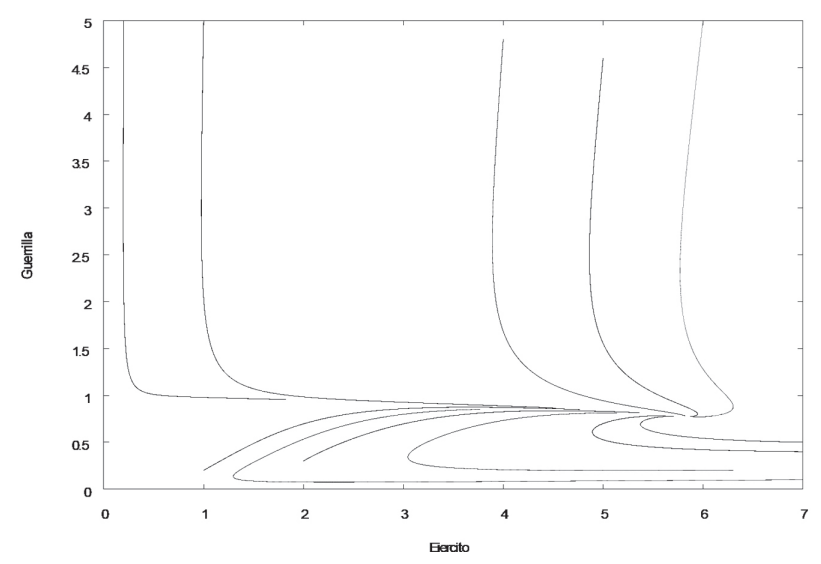

Figura 4. Espacio de fase para el conflicto Ejército-Guerrilla. En el eje horizontal número de efectivos del Ejército y en el eje vertical, número de efectivos de la Guerrilla por 25000

4. Movimiento de proyectiles de gran alcance. Es atrayente para los estudiantes analizar el movimiento parabólico, teniendo en cuenta los efectos que tiene la rotación del planeta sobre dicho movimiento. Fue así como se creó un programa basado en los efectos que la fuerza de Coriolis y la centrífuga tienen en el movimiento de un proyectil de largo alcance, esto es, un proyectil cuyo alcance máximo se encuentra entre los 35 y 50 kilómetros, y así mostrar la desviación que sufriría el obús debido a las fuerzas "fantasmas" que producen estas aceleraciones (Méndez et. al., 2009) (Figura 5).

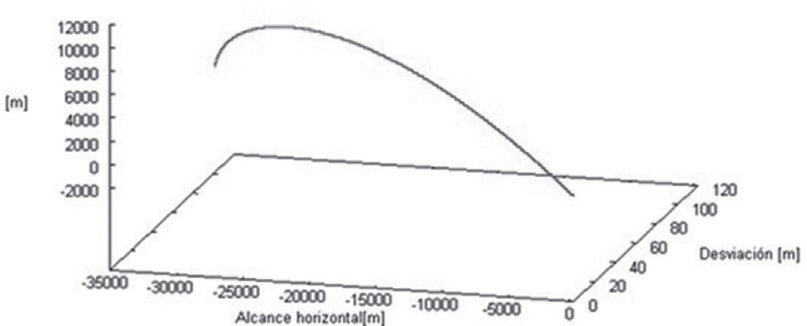

64.0000, 15.0000 scale: $1.00000,1.00000$

Figura 5. Movimiento tridimensional del misil

Los resultados de este modelo se corroboraron con algunos datos históricos que se pueden encontrar en la Web para el cañón Schwerer Gustav, utilizado por los alemanes en la Segunda Guerra Mundial; gracias a esos datos se concluyó que el modelo simula con buena aproximación el fenómeno real.

A partir de estos modelos, fue más fácil hacer el vínculo entre los códigos en C++ y PovRay, con el fin de generar animaciones de otro tipo de fenómenos físicos. Es así como se obtuvieron animaciones de situaciones físicas como:

a. Difusividad eléctrica. Se obtuvieron GIF's animados haciendo uso de la plataforma Linux-Slax, con los cuales se simularon procesos de difusión como el de una placa sometida a una diferencia de potencial de 200V entre las dos cuartas partes centrales, es decir, $1 / 4$ de la placa se encuentra sometida a un voltaje de $100 \mathrm{~V}$ y la $3 / 4$ parte de la placa posee un potencial de-100V. Así, resolviendo la ecuación de Laplace en dos dimensiones, por medio del método de diferencias finitas, se pudo crear un GIF que muestra al estudiante cómo varía el potencial a lo largo y ancho de la placa hasta que ésta llega al equilibrio (Figura 6).

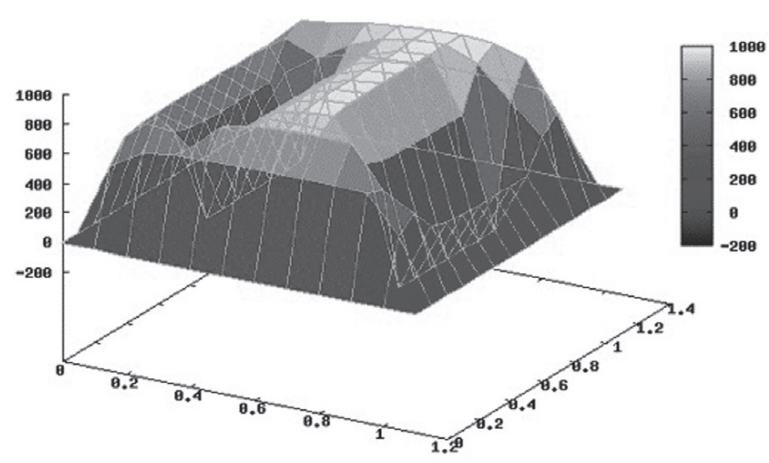

Figura 6. GIF animado del proceso de difusión en una placa, (elaborado en Linux-Slax) 
b. Oscilador anarmónico forzado. Los estudiantes también realizaron un análisis físico y matemático para un oscilador anarmónico amortiguado, el cual es un sistema conformado por una masa sujeta a un resorte y que oscila sobre un plano horizontal unidireccionalmente. Sin embargo, este sistema no cumple exactamente con la Ley de Hooke para los resortes y además se ve sometido a diversas fuerzas externas como fuerzas de fricción, de viscosidad y periódicas, que generan oscilaciones amortiguadas y forzadas, las cuales evitan que el cuerpo siga un movimiento regular y definido.

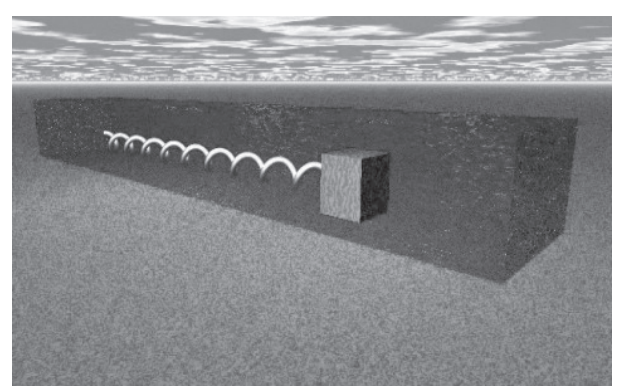

Figura 7. Fotograma de una animación desarrollada en PovRay para el caso de un oscilador anarmónico amortiguado

Debido a la dificultad de reproducir esta situación experimentalmente, los estudiantes elaboraron una animación en PovRay, la cual fue de gran utilidad para observar cómo sería el movimiento de la masa y el resorte en estas condiciones (Figura 7).

\section{Discusión}

Gracias a la buena precisión de los métodos numéricos que usaron los estudiantes para la resolución de las ecuaciones de los sistemas físicos presentados anteriormente, se pudo observar que estos modelos eran una buena aproximación a los fenómenos que podían ocurrir en realidad, luego de compararlos con algunos datos experimentales encontrados en algunas fuentes y con las predicciones de las teorías usadas. Los códigos numéricos desarrollados, traen grandes ventajas para el análisis de diferentes situaciones físicas, ya que permiten obtener muchas gráficas rápida y fácilmente para condiciones y parámetros distintos, las cuales ayudan a ampliar la comprensión de los fenómenos.

La respuesta de los estudiantes al interactuar con las modelaciones fue favorable, ya que se evidenció en ellos una mejora en los análisis que realizaban a la hora de describir los fenómenos físicos abordados. Esto se puede ver al comparar las socializaciones realizadas en grupo en el aula de clase, con la recolección de los conceptos previos, con la aplicación de la matriz de valoración y con los informes escritos que elaboraron los estudiantes luego de usar los modelos, para estudiar diferentes casos de una misma situación física.

De esta manera se encontró que los estudiantes lograban generar hipótesis coherentes y dar soluciones a los problemas que surgieron durante el proceso de modelación, gracias también a la articulación entre la estrategia y los conocimientos que ya poseían. Es interesante, notar que gracias a las visualizaciones, los estudiantes conseguían darle sentido a los datos que arrojaba el cálculo numérico del código y de esta manera lograban concluir y argumentar adecuadamente sus suposiciones.

No obstante, a pesar de que los códigos numéricos creados son fáciles de manipular y aunque la gran mayoría de los estudiantes poseen algunas bases en programación, muchos de ellos no manejan muy bien este aspecto relevante a la hora de desarrollar los modelos físicos. Lo anterior puede representar una desventaja para estos estudiantes, ya que el hecho de pensar y entender la estructura del código numérico, a partir del modelo matemático propuesto, ayuda a mejorar la comprensión del fenómeno estudiado y evita que el programa se convierta en una "caja negra", en la que el estudiante sólo tiene control de ciertos parámetros.

\section{Conclusiones}

El uso de las TIC's, tales como visualizaciones y animaciones computarizadas, en procesos de enseñanzaaprendizaje de la Física, permiten a los estudiantes elaborar modelos físicos cercanos a la realidad y con estos Ilegar a desarrollar Habilidades de Pensamiento Científico, tales como: observación, análisis, discusiónargumentación y socialización, siempre con la guía del profesor.

Si el aprendizaje depende de la creación de este tipo de modelos, de las estructuras mentales, de los procesos de pensamiento y de los intereses de los estudiantes, es necesario validar sus conceptos y/o nociones previas. Hacer esto permitirá planificar la enseñanza-aprendizaje y garantizar que ésta desarrolle y cualifique al individuo. 


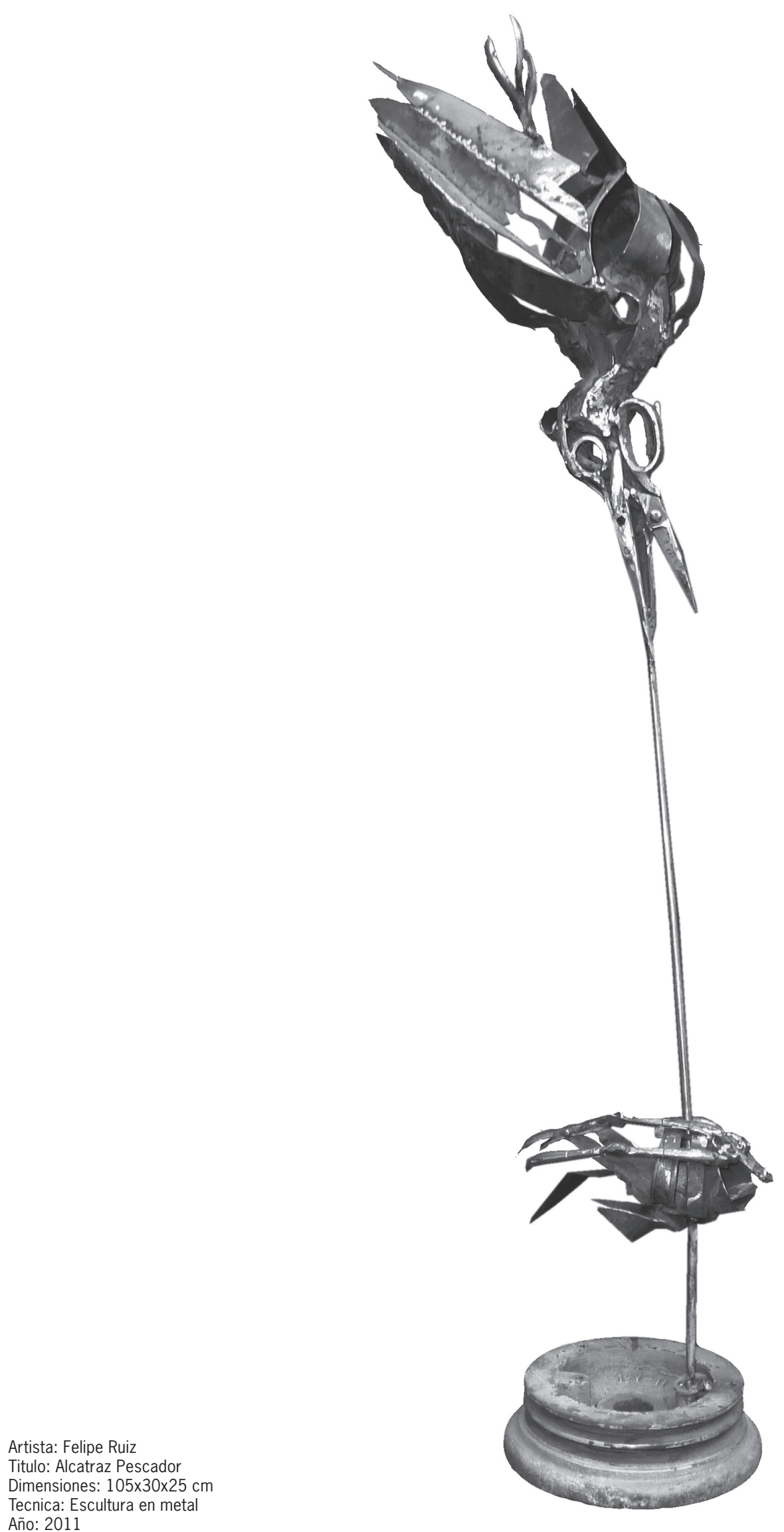


Para que las TIC's cumplan un papel formativo en la escuela, deben haber sido objeto de reflexión por parte de aquellos maestros que las utilizan, pues es el docente quien dinamiza y encamina la forma en la que los estudiantes se aproximan al conocimiento científico, lo decodifican y lo comprenden.

El uso de lenguajes de programación como $\mathrm{C}++$ y de herramientas visuales como Gnuplot y PovRay, apoyó de manera significativa el acto de aprender, ya que el análisis en tiempo real de los fenómenos bajo diferentes condiciones, les permitió evidenciar a los estudiantes situaciones inesperadas que los encaminaron a profundizar en el fenómeno, a partir de la búsqueda de explicaciones para estos hechos.

Por este motivo, dichas herramientas visuales fueron fundamentales en el escenario didáctico, Ilegando a convertirse por una parte en el centro de las explicacio- nes posteriores que se hicieron acerca de los fenómenos físicos (debido a que los análisis de estos fenómenos se obtuvieron a partir de las gráficas) y por otra, en el contexto ideal para reafirmar las comprensiones alcanzadas por los estudiantes.

\section{Agradecimientos}

Agradecemos al profesor Néstor Méndez Hincapié, del Departamento de Física de la Universidad Pedagógica Nacional, por sus grandes enseñanzas y por su valiosa orientación y colaboración en la elaboración y estudio de los modelos físicos analizados. Así mismo agradecemos al profesor William Oquendo, del Grupo de Simulación de Sistemas Físicos de la Universidad Nacional de Colombia, por su invaluable apoyo en la construcción de las animaciones realizadas con PovRay.

\section{Referencias}

Blanchard, P., Devaney, R. y Hall, G. (1999). Ecuaciones diferenciales. México: Thomson Editores, 1-3.

Chamizo, F. (2003). Modelización II: Un pase de modelos. Madrid: Universidad Autónoma de Madrid. Obtenido el 27 de Octubre, desde http://www.uam.es/fernando.chamizo, 1-4.

Edelson, D. C. y Reiser, B. J. (2006). Making Authentic Practices Accessible to Learners. En: R. K. Sawyer. (Ed.). The Cambridge Handbook of the Learning Science. Nueva York: R. Keith Sawyer, 335 -354.

Franco, A. (2008). Física con ordenador. Obtenido el 2 de Abril de 2010, desde http://www.sc.ehu.es/sbweb/fisica/

Gardner, H. (1985). The mind's new science: A history of the cognitive revolution. Nueva York: Basic Books, Inc.

González y Hernández, A. (2008). Comparación de métodos analíticos y numéricos para la solución del lanzamiento vertical de una bola en el aire. Latin American Journal of Physics Education, 2(2). Obtenido el 15 de Marzo de 2011, desde http://journal. lapen.org.mx, 170-179.

Isaza, J. y Campos, D. (2005). Modelos dinámicos de guerra: el conflicto armado. Revista de la Academia Colombiana de Ciencias, 29(110), 133-148.

Johnson-Laird, P. N. (1996). Images, Models and Propositional Representations. En: M. De Vega, M. J. Intons-Peterson, P. N. JohnsonLaird, M. Denis y M. Marschark. (Ed.). Models of Visuospatial Cognition. Oxford: Oxford University Press, 90-127.

Méndez, N., Guarín, E. y Galindo, S. (2009). El efecto Coriolis sobre el movimiento parabólico en la Tierra. Presentado en: $4^{\circ}$ Congreso Internacional sobre formación de Profesores de Ciencias (pp. 456-453), Bogotá, Colombia.

Ortiz, M. y Blanco, S. (2009). Las TIC's y su impacto en las estrategias de enseñanza en Biología. Presentado en: $4^{\circ}$ Congreso Internacional sobre formación de Profesores de Ciencias (pp. 506-512). Bogotá, Colombia.

Raviolo, A. (2009). Modelos, analogías y metáforas en la enseñanza de la Química. Revista Educación Química, 20(1), 55-60.

Sánchez, M. (2002). La investigación sobre el desarrollo y la enseñanza de las habilidades de pensamiento. Revista Electrónica de Investigación Educativa, 4(1). Obtenido el 5 de Mayo de 2010, desde http://redie.uabc.mx/vol4no1/contenido-amestoy.html, 3-10. 


\section{Diálogo del conocimiento}

Como maestro y editor de temas pedagógicos, siempre pienso en la posibilidad de que el estudiante se enfrente a su prácticas con herramientas novedosas que lo ayuden a construir el saber desde la didáctica como posibilidad de creación. Hace poco editaba una revista conocida en nuestro medio y el tema era precisamente didácticas, que difícil encontrar textos que cumpliesen con las expectativas de innovación que cambien el carácter del aula y transformen de alguna manera el modo en cómo los estudiantes aprenden, por eso la importancia de este texto ya que sirve de ejemplo no solo a los maestros de universidad, sino a los de básica y media.

El artículo muestra de una forma muy acertada el proceso de investigación, elabora una introducción precisa entorno al uso de herramientas informáticas para mejorar el aprendizaje, en este caso de estudiantes de la Licenciatura en Física de la Universidad Pedagógica Nacional, y la importancia de estas en la actual sociedad globalizada, además, con este trabajo se pretendió desarrollar Habilidades de Pensamiento Científico (HPC), acerca de este tema hacen un planteamiento no solo histórico sino teórico situando al lector en la premisa de estas habilidades y la importancia de ellas, posteriormente se desarrolla y muestra la importancia de los instrumentos computacionales como herramienta pedagógica.

Este texto es un modelo pertinente de una buena sistematización, ya que además de lo anterior se muestra la metodología muy bien desarrollada, luego la matriz con la elaboración de un cuestionario para que los estudiantes resuelvan preguntas sobre el proceso de aprendizaje, posteriormente se tabula y se dan los resultados, así se puede situar el lugar dónde se encuentran los alumnos y la investigación. Para terminar se dan las conclusiones del trabajo, siendo precisas sobre la finalidad del mismo.

Sin lugar a dudas este es un artículo bien elaborado y que muestra el trabajo que se realizó para llegar a él, unos maestros capaces y que van más allá de lo lógico y de lo que sería hacer una clase simplemente magistral, buscando aprovechar las herramientas que tienen a su alcance y así crear en sus estudiantes una nueva conciencia sobre los procesos de aprendizaje, para mí las clases no importa de que área deben llegar a estos estados, preguntémonos que necesitan nuestros alumnos y procedamos a generar didácticas, por pocos elementos que tengamos nuestra creatividad abarca espacios mayores, hagamos de nuestra clase un lugar de sueños y pasión.

Daniel Fernando Torres Páez 